\title{
Electrophysiological and Immunocytochemical Evidence for a cGMP-Mediated Inhibition of Subfornical Organ Neurons by Nitric Oxide
}

\author{
Matthias Rauch, ${ }^{1}$ Herbert A. Schmid, ${ }^{1}$ Jan deVente, ${ }^{2}$ and Eckhart Simon ${ }^{1}$ \\ ${ }^{1}$ Max-Planck-Institut für Physiologische und Klinische Forschung, W. G. Kerckhoff-Institut, 61231 Bad Nauheim, \\ Germany, and 2University of Limburg, Maastricht, The Netherlands
}

The activation of neurons in the subfornical organ (SFO) by angiotensin II (Angll) is well established and is widely regarded as the basis for the Angll-induced increase in water intake. Application of the nitric oxide (NO) donor sodium nitroprusside (SNP) led to an inhibition of the spontaneous electrical activity in $96 \%$ of the neurons sensitive for SNP $(n=50)$. In addition, the firing rate in $60 \%$ of the neurons inhibited by SNP decreased in response to superfusion with the natural substrate of the NO synthase (NOS) L-arginine whereas $70 \%$ increased their frequency after application of the NOS blocker $N^{G_{-}}$ monomethyl-L-arginine (L-NMMA; $n=10)$. The inhibitory effect of SNP could be mimicked by application of membranepermeable 8-Br-cGMP. The presence of nNOS, the neuronal isoform of NOS, was demonstrated immunocytochemically and using the NADPH-diaphorase technique on SFO slices. Using a highly selective antibody against cGMP in formaldehydefixed tissue, the NO donors SNP, 3-morpholinosydnonimine (SIN-1), and S-nitroso- $N$-acetyl-DL-penicillamine (SNAP) caused a strong increase in cGMP formation when applied under the same conditions as used for the electrophysiological recordings. These electrophysiological results suggest an important role for NO in SFO-mediated responses and offer a plausible explanation for the in vivo-observed opposite effects of Angll and NO on water intake.

Key words: sodium nitroprusside; nitric oxide; thirst; drinking; neuron; osmoregulation; rat; electrophysiology; subfornical organ; angiotensin II
The subfornical organ (SFO) represents one of the neural circumventricular organs (CVO) lacking a blood-brain barrier and has been implicated as a key structure in osmoregulation (McKinley et al., 1990). One of the best characterized functions of the SFO of mammals is the induction of drinking in response to bloodborne angiotensin II (AngII) (Fitzsimons, 1979). The dipsogenic effect of AngII has been shown to correlate with an increase in electrical activity of the majority of SFO neurons in response to AngII in mammals and birds (Fitzsimons, 1980; Phillips, 1987; Simon et al., 1992). Electrical stimulation of the SFO results in an increase in water intake (Smith et al., 1995) and elevated blood pressure (Ishibasi and Nicolaidis, 1981; Ferguson et al., 1984) in rats. Conversely, it has been shown that the direct application of the nitric oxide (NO) donor sodium nitroprusside (SNP) to the SFO by microapplication inhibits the AngII-induced water intake in rats (Nicolaidis and Fitzsimons, 1975). Autoradiographical studies have demonstrated the presence of receptors for AngII in the SFO of rats (Mendelsohn et al., 1984), and histochemical studies have shown that neuronal NO synthase (nNOS) is present in all CVOs, including the SFO in large quantities, using immunocytochemical and NADPH-diaphorase techniques (Jurzak et al., 1994). These results led to the suggestion that the activity of

\footnotetext{
Received Aug. 30, 1996; revised Oct. 11, 1996; accepted Oct. 21, 1996.

This work was supported by Grant Si 230/8-1 from the Deutsche Forschungsgemeinschaft. We greatly appreciate the help and technical assistance of G. Jurat.

These experiments comply with Principles of Animal Care, publication No. 85-23, revised 1985, from National Institutes of Health and also with the current laws of the country in which the experiments were performed.

Correspondence should be addressed to Dr. Herbert A. Schmid, Max-PlanckInstitut für Physiologische und Klinische Forschung, W. G. Kerckhoff-Institut, Parkstrasse 1, 61231 Bad Nauheim, Germany.

Copyright (C) 1996 Society for Neuroscience $0270-6474 / 96 / 170363-09 \$ 05.00 / 0$
}

SFO neurons that were excited by AngII might also be affected by locally released NO.

The aim of this study was to investigate the possible effect of nitric oxide on the electrical activity of spontaneously active neurons in the rat SFO in a slice preparation. In a similar investigation on slices of the rat spinal cord (Pehl and Schmid, 1997), SNP was shown to be a potent and specific NO donor on the same experimental setup and was also used, therefore, in this study to investigate the effect of NO. Based on the assumption that NOS is present and still functioning in our in vitro slice preparation, increasing or decreasing the production of endogenous NO, by superfusing the slice with L-arginine, the natural substrate of the NOS, or with $N^{\mathrm{G}}$-monomethyl-L-arginine (L-NMMA), a selective blocker of the NOS, should result accordingly in the same or opposite effects as caused by the NO donor.

In an immunocytochemical study using an antibody that was specifically designed to recognize cGMP in paraformaldehydefixed tissues (deVente et al., 1989; deVente and Steinbusch, 1992) the NO-dependent production of cGMP was investigated, because cGMP is known to be the second messenger formed by the NO-dependent activation of soluble guanylyl cyclase (Murad, 1994; Garthwaite and Boulton, 1995). Using the same in vitro slice preparation as for the electrophysiological recordings, the amount of cGMP produced was examined after incubating the SFO slices for 10 min with three different NO donors. To determine whether the SFO neurons that possibly produce NO are identical to the neurons that respond to it, immunocytochemical double labeling was performed on identical sections using antibodies against NOS as well as cGMP.

The possible neuronal relevance of an NO-induced increase in cGMP content in the SFO was assessed electrophysiologically by 
applying the membrane-permeable analog 8-Br-cGMP on neurons that showed responsiveness to $\mathrm{NO}$, and the resulting change in activity was compared to the effect of the NO donor on identical cells.

\section{MATERIALS AND METHODS}

The materials and methods were, with minor modifications, the same as described previously (Schmid and Simon, 1992). Briefly, adult male Wistar rats (180-270 gm) were decapitated, and their brains were quickly removed and superfused with ice-cold artificial CSF (aCSF) of the following composition (in $\mathrm{mm}$ ): $\mathrm{NaCl} 124, \mathrm{KCl} 5, \mathrm{NaH}_{2} \mathrm{PO}_{4} 1.2, \mathrm{MgSO}_{4} 1.3$, $\mathrm{CaCl}_{2} 1.2, \mathrm{NaHCO}_{3} 26$, glucose $10, \mathrm{pH}: 7.4$, equilibrated with $95 \% \mathrm{O}_{2} / 5 \%$ $\mathrm{CO}_{2}, 290 \mathrm{mOsm} / \mathrm{kg}$. The brain was trimmed to a square block containing the entire hypothalamus, from which a coronal section was cut by hand at the level of the anterior commissure. A slice of the body of the fornix, containing the entire SFO, was cut by hand and preincubated in aCSF at $35^{\circ} \mathrm{C}$ for $1 \mathrm{hr}$. Slices were transferred to the recording chamber and fixed to the bottom of the chamber with a small metal weight. The gold-plated recording chamber was made from solid brass and, when perfused with aCSF, contained a fluid volume of $\sim 0.7 \mathrm{ml}$. The chamber was constantly perfused with aCSF at a rate of $1.6 \mathrm{ml} / \mathrm{min}$. ACSF entering the recording chamber was prewarmed to the same temperature as the solution already present in the chamber. The temperature was kept constant at $37^{\circ} \mathrm{C}$ using a Peltier element. Extracellular recordings were made from SFO neurons using glass-coated platinum-iridium electrodes. The SFO could easily be identified by its protrusion into the third ventricle and the lateral blood vessels lining the organ on both sides. AngII (Sigma, Deisenhofen, Germany) and SNP (Schwarz Pharma, Monheim, Germany) were added to the aCSF shortly before the application. SNP stock solutions were prepared fresh on the experimental day from preweighed vials, stored on ice, and protected from light until use. After a stable recording from a single neuron had been established, its responsiveness was tested by switching to a perfusion solution containing the drug under consideration. The recorded action potentials were amplified and displayed on a storage oscilloscope (Gould, Germany) and were analyzed, after passing a window discriminator (World Precision Instruments), with custommade software (Spike2 from Cambridge Electronic Design) on a personal computer.

Normally $10 \mathrm{ml}$ of aCSF containing the drug was superfused per stimulus, except for experiments on the stimulation or inhibition of NOS, in which $20 \mathrm{ml}$ was used. The concentrations of AngII $\left(10^{-8}\right.$ to $\left.10^{-7} \mathrm{M}\right)$ were chosen according to previous experiments (Schmid and Simon, 1992) showing that this concentration induced clearly visible responses with minimum desensitization. SNP was used at a concentration between $10^{-6}$ and $10^{-3}$ M. 8-Br-cGMP (Sigma), L- and D-NMMA (Alexis, Grünberg, Germany), and L- and D-arginine (Sigma) were applied at a concentration of $10^{-3} \mathrm{M}$. From the continuously recorded rate meter counts, the average discharge rate of each neuron was evaluated for $60 \mathrm{sec}$ before the stimulus. This value (referred to as "control") was subtracted from all subsequent changes in firing rate, and the results were expressed as "percent change of control." The latency of a response comprised the time between the occurrence of the drug in the recording chamber until the start of the excitatory or inhibitory responses. If the averaged change of discharge rate during the entire response time was reversibly larger than $\pm 20 \%$, the neuron was considered sensitive to the applied substance; if no obvious change in the spontaneous activity was observed, the firing rate was averaged over a $500 \mathrm{sec}$ period after the drug entered the recording chamber and compared to the control values. Mean values in the text are given with SEM.

For the immunohistological study, the preparation of the SFO was the same as described for the electrophysiological recordings. The SFO slices were incubated for $40 \mathrm{~min}$ in $2 \mathrm{ml}$ of aCSF $+1 \mathrm{~mm} 3$-isobutyl-1methylxanthine (IBMX; Sigma) at $37^{\circ} \mathrm{C}$ placed in multiwell tissue culture chambers under an atmosphere of $5 \% \mathrm{CO}_{2} / 95 \% \quad \mathrm{O}_{2}$. The $\mathrm{NO}$ donors SNP, SNAP (Alexis), and SIN-1 (Cassella, Frankfurt, Germany) were added for the last $10 \mathrm{~min}$ to a final concentration of $10^{-4} \mathrm{M}$. The incubation was terminated by adding $2 \mathrm{ml}$ of an ice-cold fixative solution (final concentration $4 \%$ formaldehyde in PBS for $1 \mathrm{hr}$ at $4^{\circ} \mathrm{C}$ ), followed by $2 \mathrm{hr}$ post-fixation in cold PBS (containing 4\% formaldehyde and $10 \%$ sucrose) and, finally, washed for $30 \mathrm{~min}$ in PBS $+10 \%$ sucrose. Immediately thereafter, $10-\mu \mathrm{m}$-thick sections were cut on a cryostat at $-20^{\circ} \mathrm{C}$, thawed on poly-L-lysine-coated slides, and processed immediately for immunocytochemistry. The slides were air-dried for $20 \mathrm{~min}$ at room temperature, rehydrated, and washed three times for 5 min each in PBS.
The sections were incubated overnight at $4^{\circ} \mathrm{C}$ with cGMP antiserum (deVente et al., 1989) from sheep [1:6000 in PBS $+0.5 \%$ Triton X-100 (PBS-T)] and nNOS antiserum (Alexis) from rabbit (1:1000 in PBS-T). The next day, sections were washed three times for 5 min each in PBS and incubated for $75 \mathrm{~min}$ at room temperature with FITC-conjugated donkey anti-sheep immunoglobulins and Cy3-conjugated goat anti-rabbit immunoglobulins, respectively. For the double-labeling experiments, the incubation procedures were done separately in the following order: cGMP antibody from sheep, nNOS antiserum from rabbit, Cy3-conjugated goat anti-rabbit immunoglobulins, and FITC-conjugated donkey anti-sheep immunoglobulins.

Finally, the slides were washed three times for 15 min each in PBS and mounted in PBS/glycerol (1:1 v/v; Cityfluor). Photographs were taken on a Zeiss Axioskop using a Kodak Ektachrome 400 film, with an adjustment of $1600 \mathrm{ASA}$ at the camera.

The NADPH-diaphorase staining was performed as described previously for rats (Jurzak et al., 1994), i.e., the slices were the same as already used for the immunocytochemistry, thus allowing a direct comparison of nNOS and NADPH-diaphorase staining on identical sections. Briefly, the sections containing the SFO were washed three times for $5 \mathrm{~min}$ each in phosphate buffer (PB), $\mathrm{pH} 7.4$, and then incubated for $2 \mathrm{hr}$ in $0.1 \mathrm{M} \mathrm{PB}$, pH 8.0, containing $55 \mu \mathrm{M}$ NADPH, $0.12 \mathrm{~mm}$ nitroblue tetrazolium, and $0.3 \%$ Triton X-100 (all from Sigma) at $37^{\circ} \mathrm{C}$ in the dark. After the incubation, sections were rinsed in $\mathrm{PB}, \mathrm{pH} 7.4$, air-dried, and coverslipped with Entellan (Merck, Darmstadt, Germany). Photographs were taken on a Zeiss Axioskop using Kodak Ektachrome 400 film.

\section{RESULTS \\ Immunocytochemical study and NADPH-diaphorase staining}

The level of cGMP in the rat SFO after different treatments is illustrated by the brightness of the immunocytochemical staining in Figure $1 a-d$. Each of the four SFOs from which these sections were taken was treated the same except for the incubation with the NO donors. Figure $1 a$ shows the coronal section of a rat SFO, which was not incubated with an NO donor and thus represents the control level of cGMP present in this tissue. After $10 \mathrm{~min}$ incubation with the NO donor SNP $\left(10^{-4} \mathrm{M}\right.$; Fig. $\left.1 b\right)$ or SNAP $\left(10^{-4} \mathrm{M}\right.$; Fig. $\left.1 c\right)$, the cGMP immunoreactivity was increased. The increase in cGMP label was not restricted to neuronal somata, but could also be observed in fibers and non-neuronal (ependymal) cells throughout the SFO. In line with previous experiments on spinal cord slices (Pehl and Schmid, 1996), SIN-1 (Fig. 1d) was the least effective NO donor and caused at equimolar concentrations only a small increase in cGMP content.

Microfluorometrical measurements of a coronal section of the medial SFO, which had been treated for 10 min with SNP, calculated a $209 \%$ increase in cGMP content (normalized to the surface area) as compared to the control (Fig. 1a,b).

The distribution of the cGMP immunoreactivity was not uniform throughout the SFO. With the exception of the rostral part of the SFO, in which the cGMP immunoreactivity was evenly distributed over the entire section, the medial and caudal part of the SFO displayed less cGMP fluorescence in the central region as in the more lateral parts of the SFO. This regional difference was the same in the controls and in SFO slices that had been treated with an NO donor, although in the treated tissues these regional differences were more apparent (Fig. $1 a-d$ ). Figure $1, e$ and $f$, shows high-power magnifications of the same cross section of the ventral part of the SFO that contacts the third ventricle. In this section, a double labeling for nNOS (Fig. 1e) and cGMP (Fig. 1f) was performed, and it showed that nNOS and cGMP are codistributed in the same regions of the SFO; but on the cellular level, the nNOS and cGMP staining was almost never colocalized in identical cells. The large arrows point at two cells that showed 

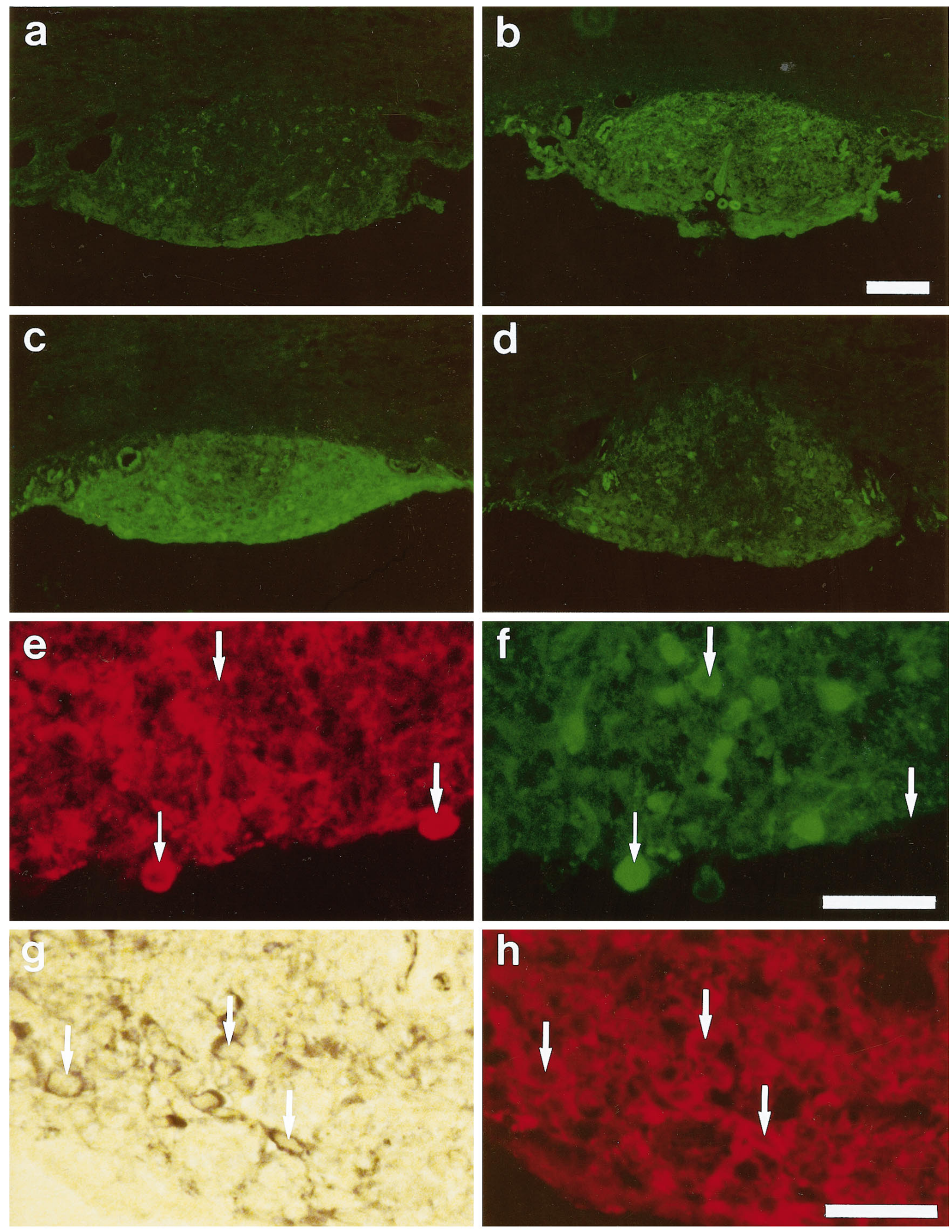

Figure 1. Immunocytochemical and NADPH-diaphorase staining of 10- $\mu$ m-thick sections of the rat subfornical organ (SFO). $a-d$, Comparison of cGMP immunoreactivity after normal treatment (control, $a$ ) and after 10 min incubation with sodium nitroprusside (SNP; $b$ ), $S$-nitroso- $N$-acetylDL-penicillamine (SNAP; $c$ ), and 3-morpholinosydnonimine (SIN-1; $d$ ). Markedly increased levels of cGMP are visible after treatment with NO donors. Scale bar (shown in $b$ ): $200 \mu \mathrm{m} . e, f$, Double labeling of an identical SFO section for neuronal NO synthase (nNOS; $e$ ) and cGMP $(f$ ) immunoreactivity. One cell labeled by both antibodies is marked with a small arrow, whereas the large arrows mark cells exclusively stained by the cGMP antibody or nNOS antibody, indicating a codistribution rather than a colocalization. Scale bar (shown in $f$ ): $40 \mu \mathrm{m} . g, h$, Colocalization of NADPH-diaphorase staining $(g)$ with nNOS immunoreactivity $(h)$ in an identical section of the SFO. Examples of cells labeled by both techniques are marked with arrows. Scale bar (shown in $h$ ): $40 \mu \mathrm{m}$. 
either a strong NOS or a strong cGMP staining, although very rarely double labeled cells could also be observed (small arrow).

Figure $1 g$ shows an NADPH-diaphorase staining of the same section of the SFO, in which the NOS had previously been visualized immunocytochemically, using an antibody against the neuronal isoform of the NOS (Fig. 1h). NOS-immunopositive neurons could be seen in the SFO, clearly distinguished from unlabeled cells in the surrounding tissue, indicating the presence of nNOS in the rat SFO. Immunolabeling as well as NADPHdiaphorase staining was achieved in cell bodies and axonal and/or dendrite processes with a complete colocalization (see arrows for cells marked with both techniques). The brown/blue color and the staining intensity of the NADPH-diaphorase were similar to the labeling in other brain regions such as the cerebellum, known to contain nNOS, but it was clearly different from the labeling that occurred in hypothalamic regions, including the supraoptic nucleus (SON), nucleus paraventriculus (PVN), and scattered neurons in the cortex, where individual neurons showed heavy and black staining.

\section{Electrophysiological study}

Only those neurons that showed a relatively stable spontaneous activity and that could be tested for their responsiveness to AngII as well as SNP were included in these results. Furthermore, the effects of both agents had to be reversible to be considered for quantitative evaluation. The mean spontaneous frequency of all 95 neurons tested was $5.5 \pm 0.2$ spikes/sec. With respect to the AngII responsiveness of the investigated sample of neurons, $83 \%$ $(n=95)$ were excited and the remaining neurons were unresponsive, i.e., not a single neuron was inhibited. The excitatory effect of AngII was visible after a latency of $61 \pm 5 \mathrm{sec}$, produced a mean increase averaged from the beginning to the end of the response in firing rate of $3.0 \pm 0.2 \mathrm{~Hz}$ (i.e., $54 \%$ ), and had a duration of $436 \pm 19 \mathrm{sec}$.
Table 1. Summary of the effects of AngII and SNP on neurons of the rat subfornical organ

\begin{tabular}{lccc} 
& AngII excitation & AngII no effect & Total \\
\hline SNP inhibition & 40 & 8 & 48 \\
SNP no effect & 37 & 8 & 45 \\
SNP excitation & 2 & 0 & 2 \\
\hline Total & 79 & 16 & 95 \\
\hline
\end{tabular}

Fifty-one percent of the investigated neurons $(n=95)$ were inhibited by SNP when applied at concentrations of $10^{-5}$ to $10^{-3} \mathrm{M}$. Only $2 \%$ of the neurons were excited, and the remaining $47 \%$ were unaffected. For the inhibitory effect, we obtained a mean decrease, averaged from the beginning to the end of the response in the discharge rate, of $-1.8 \pm 0.1 \mathrm{~Hz}$ (i.e., $-32 \%$ ) with a $110 \pm 18 \mathrm{sec}$ latency and a $464 \pm 23 \mathrm{sec}$ duration. Eighty-three percent of the neurons that were inhibited by SNP reacted to AngII with an increase in activity (Table 1). Figure 2 shows the continuous activity of a rat SFO neuron that was tested consecutively with both substances. The spontaneous activity increased rapidly in response to $10^{-7} \mathrm{M}$ AngII and decreased asymptotically to the previous frequency after the end of the application. Superfusion with SNP $\left(10^{-4} \mathrm{M}\right)$ reversibly decreased the firing rate of the same neuron, also with a rapid onset and a slow recovery to the base frequency. It could also be shown that the AngII-induced excitation could be antagonized by SNP and that the SNP-induced inhibition could be antagonized by AngII. Figure $3 a$ is an example of a neuron that was reversibly inhibited by SNP. Although this neuron did not generate action potentials anymore in response to SNP, superfusion with AngII in the continuous presence of SNP caused a long-lasting excitation of this cell. In a different neuron, the inhibitory effect of SNP, which was weak under control conditions, became even more pronounced in this neuron in the

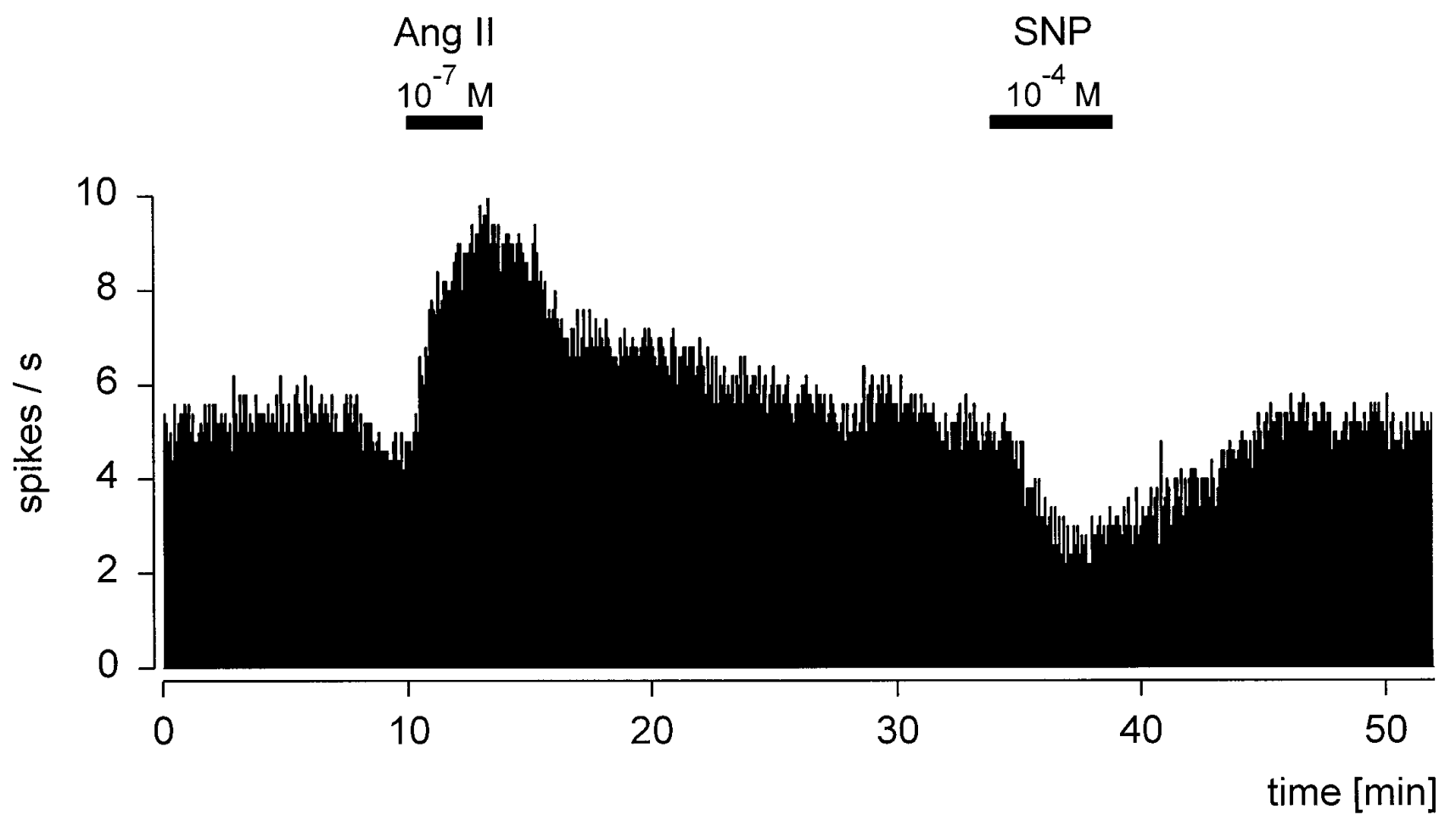

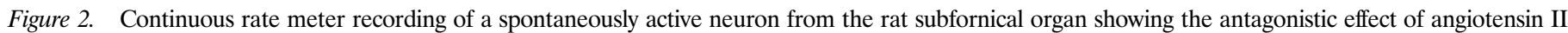

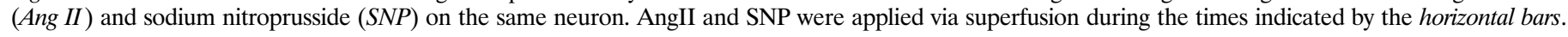



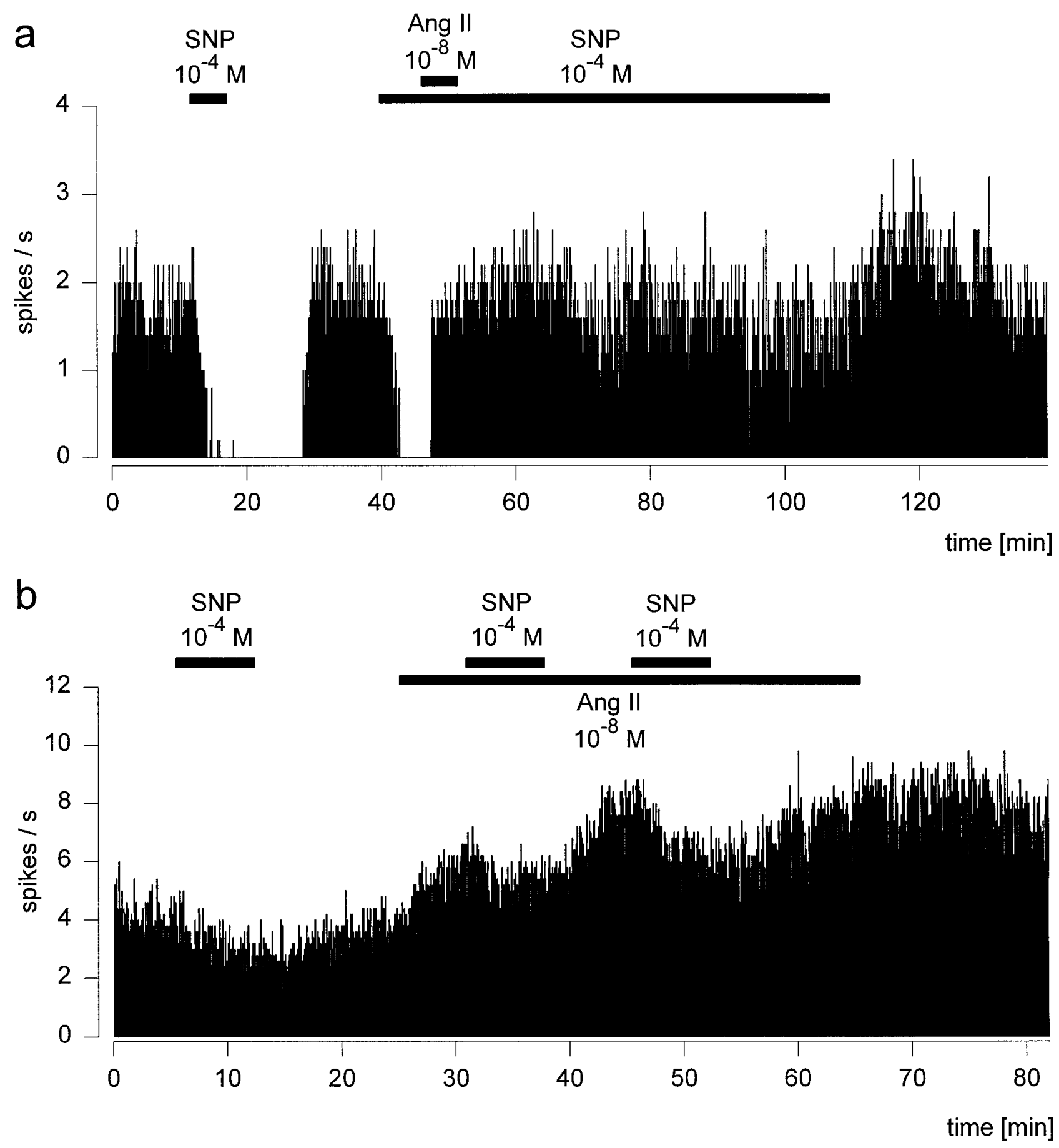

Figure 3. $a$, The inhibitory effect of sodium nitroprusside $(S N P)$ on this neuron could be antagonized by angiotensin II (Ang II) in the presence of the NO donor. $b$, Repeated application of SNP was able to antagonize reversibly the excitatory effect of continuously applied AngII on this neuron.

continuous presence of AngII, which caused the usual excitatory response (Fig. 3b). Qualitatively similar results were obtained in each of the 4 other neurons tested.

The inhibition of the discharge rate by SNP, examined in a range between $10^{-6}$ and $10^{-3} \mathrm{M}$, was dose-dependent (Fig. 4). The threshold concentration of SNP causing an effect was $10^{-5} \mathrm{M}$, as illustrated by the inset. This relationship is obvious in the continuous rate meter recording, which indicates that higher concentrations of SNP were able to cause reductions in the firing rate to 0 spikes/sec. Additionally, this neuron was excited by superfusion with AngII $\left(10^{-8} \mathrm{M}\right)$.

The question of whether the inhibitory response seen after superfusion with SNP was specific to NO was addressed further by activating or inactivating the production of endogenous $\mathrm{NO}$ via activation or inhibition of the endogenous NOS. This set of experiments was based on the assumption that endogenous NOS was still active under our in vitro slice conditions and that this enzyme produced sufficient amounts of NO to affect the firing rate 


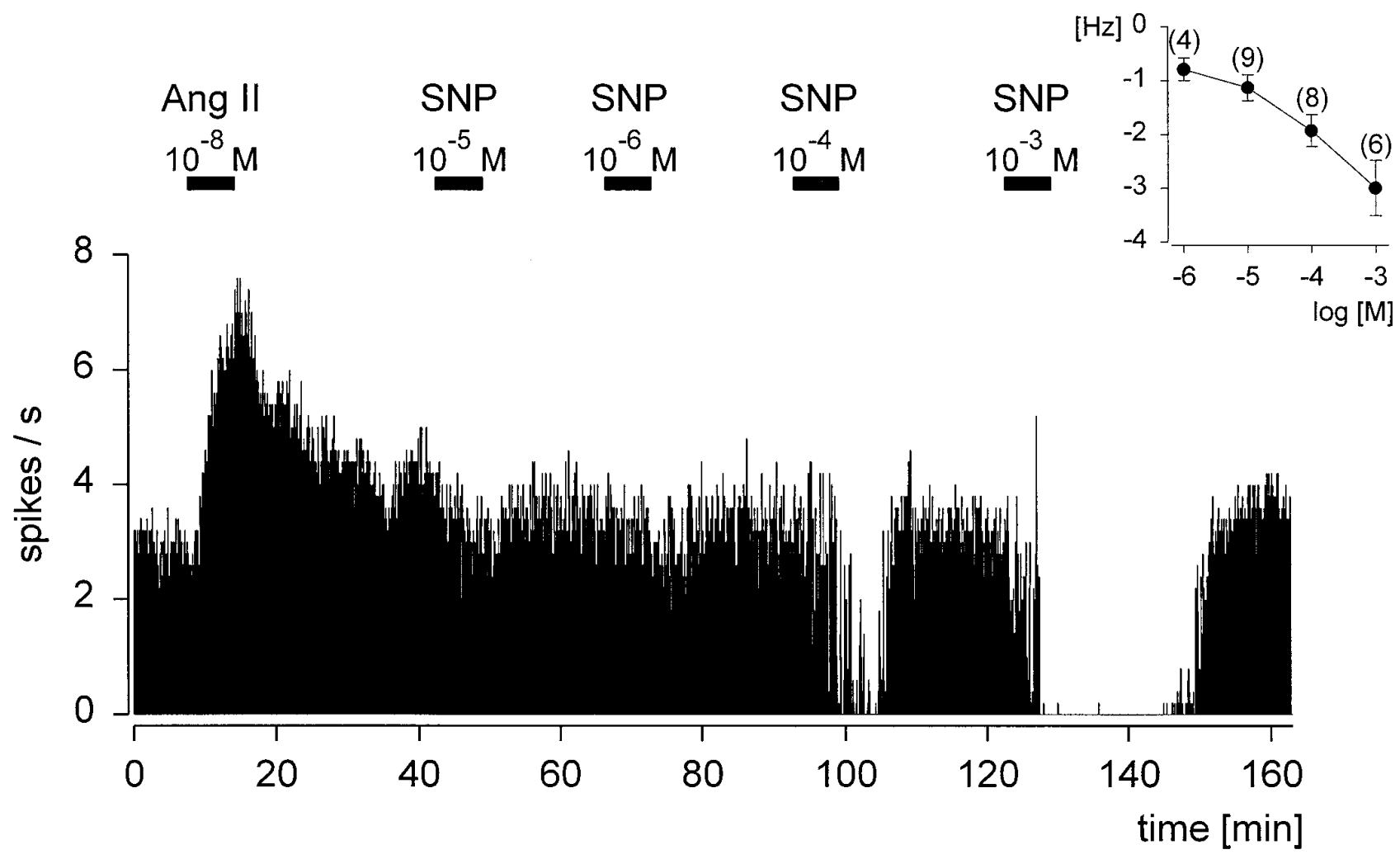

Figure 4. Dose-dependent effect of sodium nitroprusside (SNP) on an angiotensin II (Ang II)-sensitive neuron, which was consecutively superfused during the times indicated by the horizontal bars. The inset shows the averaged dose-response curve of the inhibitory effect of SNP on rat subfornical organ neurons.

of neurons located in the vicinity of the NO-producing cells. As shown in Figure 5, superfusion with the NOS blocker L-NMMA $\left(10^{-3} \mathrm{M}\right)$ increased the firing rate of the neuron, in contrast to the inhibitory effect of the natural substrate of the NOS (L-arginine, $\left.10^{-3} \mathrm{M}\right)$. A decrease in the firing rate by L-arginine on neurons that were inhibited by SNP was obtained in 6 out of 10 cases, with the other 4 neurons showing no effect. With respect to the mean response frequency change, the quality of the inhibition by L-arginine was not significantly different from that caused by SNP for the same neurons (paired $t$ test, $p=0.33$ ). Blocking the NO-producing enzyme with L-NMMA caused an excitation in $70 \%$ of the SNP-sensitive neurons, and this effect could be reversed by the application of L-arginine. The biologically inactive D-enantiomers of the NOS blocker (D-NMMA, $10^{-3} \mathrm{M}$ ) and the NOS substrate (D-arginine, $10^{-3} \mathrm{M}$ ) had no effects on the discharge rate of each of the neurons examined $(n=6)$ that showed responsiveness to the respective L-forms.

Superfusion of 8 -Br-cGMP $\left(10^{-3} \mathrm{M}\right)$, a membrane-permeable analog of cGMP, mimicked the inhibitory effect in 8 out of 13 SNP-sensitive neurons, whereas the 5 remaining neurons were not affected. The continuous rate meter recording of a rat SFO neuron, which was reversibly inhibited by SNP $\left(10^{-3} \mathrm{M}\right)$ and also reversibly inhibited by 8 -Br-cGMP $\left(10^{-3} \mathrm{M}\right)$, is shown in Figure 6. The statistical comparison between the mean inhibitory effects of both substances applied at identical effective concentrations to the same neurons $(n=8)$ showed no significant differences (paired $t$ test, $p=0.88)$.

\section{DISCUSSION}

The majority of rat SFO neurons responded to an application of AngII with an increase in electrical activity. Not a single neuron was inhibited by AngII, confirming previously published electrophysiological studies using the same preparation (Schmid and Simon, 1992; $\mathrm{Li}$ and Ferguson, 1993). This result strongly argues for a direct action of AngII on the majority of SFO neurons and against a major inhibitory interaction of AngII-sensitive neurons and other neurons within the rat SFO. Functionally, this excitation is regarded as the neuronal basis for the AngII-induced increase in water intake (Felix and Akert, 1974; Phillips and Felix, 1976).

In vivo studies on the effects of AngII and the NO donor SNP applied directly to the SFO have demonstrated the inhibitory effect of SNP on the AngII-induced water intake (Nicolaidis and Fitzsimons, 1975). Central application of the NOS substrate L-arginine into the fourth ventricle or into the preoptic area also reduced the AngII-induced water intake (Calapai et al., 1992, 1994), although presumably this response did not involve the SFO.

Extrapolating from the well characterized effect that the AngIIinduced water intake is attributable to an excitatory effect of this peptide on the activity of SFO neurons (Felix and Akert, 1974; Phillips and Felix, 1976; Simon et al., 1992), a substance that inhibits SFO-mediated water intake should accordingly have an inhibitory effect on neuronal activity, possibly on the same cells. Focusing only on the neurons that were excited by AngII, our electrophysiological results demonstrated that $95 \%$ of the SNPresponsive neurons were inhibited by the NO donor. Furthermore, it could be shown that AngII and SNP have antagonistic effects on the same neurons, because the AngII-induced excitation could still be observed during the inhibitory action of continuously applied SNP and the inhibitory effect of SNP could still be observed during an ongoing excitatory effect of AngII. Therefore, the opposite and antagonistic effects of AngII and SNP on the 


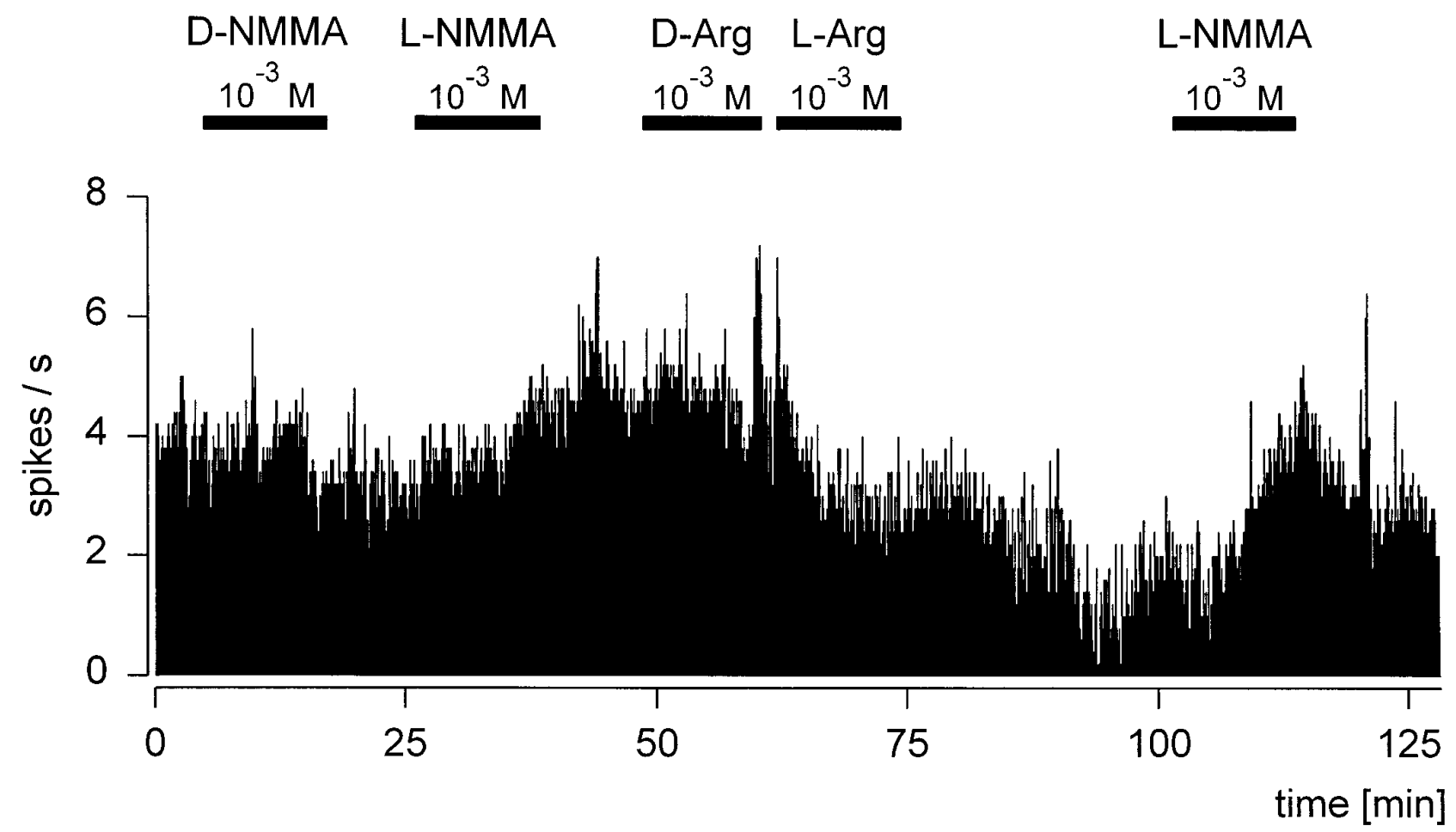

Figure 5. Continuous rate meter recording of a spontaneously active neuron from the subfornical organ, demonstrating the effects of the natural substrate for NOS L-arginine $(L-A r g)$, the false substrate $N^{\mathrm{G}}$-monomethyl-L-arginine $(L-N M M A)$, and their biologically inactive D-enantiomers $(D$ - $A r g$, $D-N M M A)$, which serve as controls. The horizontal bars represent the times of the respective superfusion.

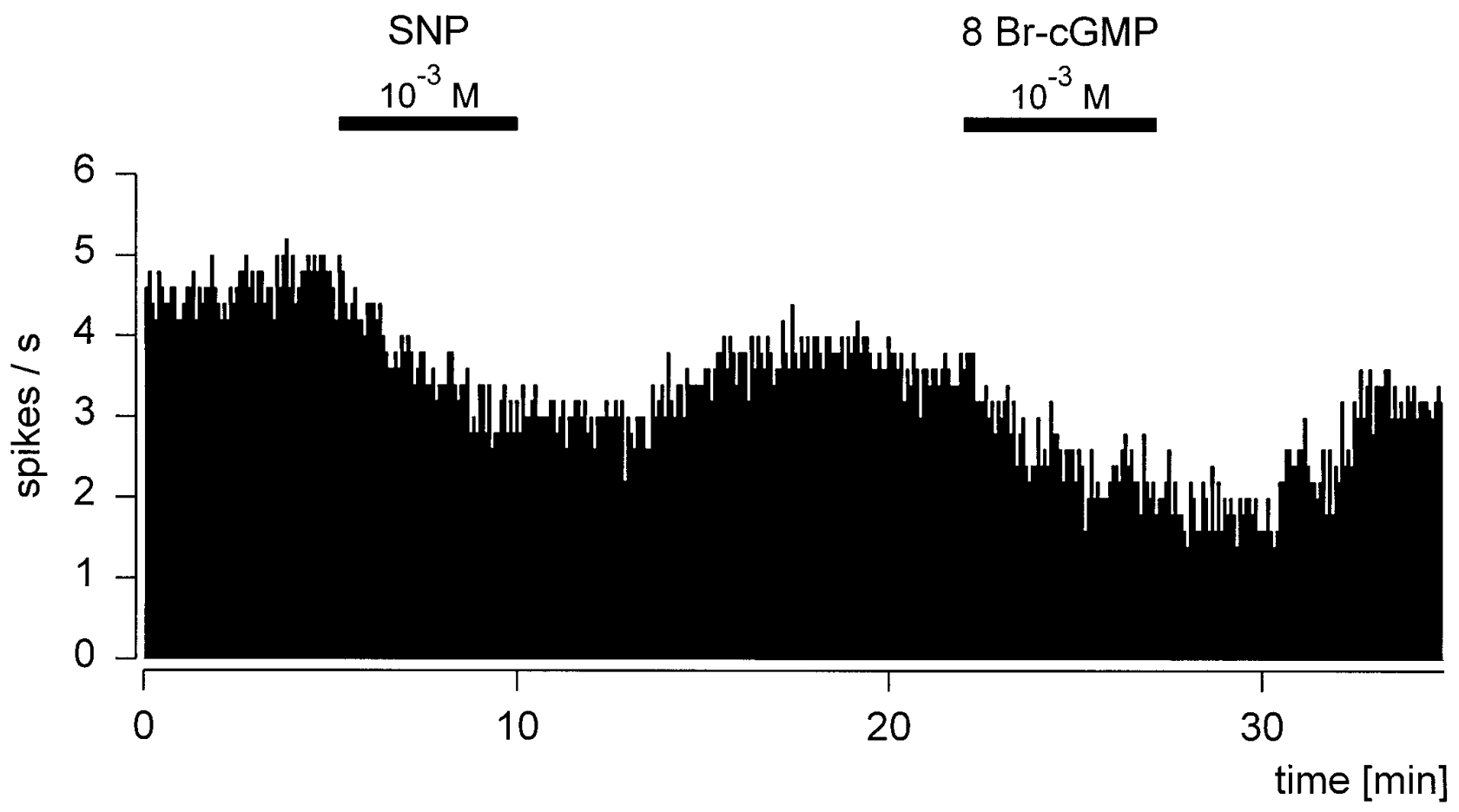

Figure 6. Continuous rate meter recording of a spontaneously active neuron from the rat subfornical organ. During the times indicated by the horizontal bars, sodium nitroprusside $(S N P)$ or the membrane-permeable analog 8-Br-cGMP $(8 \mathrm{Br}$-cGMP) was applied via superfusion. 8-Br-cGMP mimics the inhibitory effect of the NO donor SNP on the same neuron.

electrical activity of largely identical SFO neurons might represent the cellular basis for the antagonistic effects observed on water intake.
The fact that NADPH-diaphorase-positive neurons are present in the rat SFO stresses the functional relevance of the electrophysiological results. Hope et al. (1991) showed the identity of 
neuronal NADPH-diaphorase and NOS in many central neurons, and Jurzak et al. (1994) demonstrated a colocalization of NADPH diaphorase reaction product and immunohistological nNOS antibody staining in the rat SFO. In addition, the NOS is still functionally active in our slice preparation as demonstrated by the inhibitory effect of the NOS substrate L-arginine and the activation of SNP-sensitive neurons in response to the NOS blocker L-NMMA. The specificity of these results is underlined by the ineffectiveness of the biologically inactive D-enantiomers. These in vitro results strongly suggest that the neuronal activity of SFO neurons, and thus the SFO-mediated water intake, is inhibited by endogenous NO that is produced and released within the SFO itself.

With regard to the transduction mechanism of NO, it is known that NO activates the soluble form of the guanylyl cyclase in many neuronal systems (Murad, 1994; Garthwaite and Boulton, 1995), leading to an increased intracellular concentration of cGMP. A strong NO-induced elevation of cGMP levels could be shown in rat SFO slice preparations using an antibody that detects cGMP in fixed tissues. The second messenger cGMP influences neuronal activity via direct or indirect mechanisms involving different membrane channels (Garthwaite and Boulton, 1995) and has been shown recently to cause excitatory as well as inhibitory responses in different laminae of spinal cord slices (Pehl and Schmid, 1997). Therefore, using the membrane-permeable analog 8-Br-cGMP, we could mimic the inhibitory effect of SNP on neurons in the rat SFO, providing electrophysiological evidence for the involvement of cGMP in the inhibitory signal transduction pathway of NO in the SFO.

Biochemical data showed that the increased intracellular $\mathrm{Ca}^{2+}$ concentration that is necessary to activate the NOS would simultaneously inhibit any guanylyl cyclase activity (Vincent and Hope, 1992), which also suggests that cells containing NOS are presumably not identical to cells that respond to NO with an increase in cGMP content. This hypothesis has been confirmed by showing that, despite the fact that NADPH-diaphorase-positive and cGMP-positive cells were located in the same regions of the rat brain, the NADPH-diaphorase-positive cells and cGMP-positive cells were complementary rather than identical (Southam and Garthwaite, 1993). This codistribution rather than colocalization of nNOS and cGMP was also demonstrated in this study in the rat SFO, by showing that NADPH-diaphorase and nNOS-positive cells were identical, whereas cGMP-positive cells were located in close proximity, but with very few exceptions never identical, to these cells.

Based on these data, it can be speculated that the spontaneously active neurons in the rat SFO that are responsive to AngII as well as SNP are presumably not identical with the NADPHdiaphorase-positive neurons, but are located in the vicinity of those cells. This means that AngII-sensitive neurons in the rat SFO are subject to inhibition by NO via the release of NO from adjacent NOS-containing neurons.

The question that physiological stimulus triggers the release of $\mathrm{NO}$ within the SFO in vivo has not been addressed so far, although it can be predicted that it should be an antidipsogenic stimulus and/or an inhibitory stimulus on AngII-sensitive neurons in the rat SFO. Atrial natriuretic factor (ANF) has been shown to cause effects that are generally opposite to the actions of AngII (Kleinert et al., 1984; Harris et al., 1987; Saavedra, 1992; Wright and Harding, 1992), including an antagonistic effect on AngII-induced water intake (Ehrlich and Fitts, 1991) and an inhibitory effect on SFO neurons of rats but not ducks (Hattori et al., 1988; Schmid and Simon, 1992; Schmid et al., 1995). Immunocytochemical evidence showing that ANF (deVente et al., 1989) as well as NO (this study) can increase cGMP production in the SFO and the fact that the ANF-induced increase in cGMP levels in the immature rat brain can be partly blocked by an NOS inhibitor (deVente and Steinbusch, 1992) are compatible with the notion that ANF, in addition to its receptor-intrinsic guanylyl cyclase activity, could possibly inhibit SFO neurons indirectly via the release of NO within the SFO.

In summary, these results suggest that NO is a major inhibitory modulator in the rat SFO and that its inhibitory effect is mediated by NO that is produced and released within the SFO under as yet unknown physiological conditions. It is suggested that the inhibitory effect of NO depends on the elevation of cGMP in SFO neurons that are generally not identical to the cells producing NO. Functionally, these results imply that the inhibitory effect of NO on AngII-sensitive neurons in the rat SFO offers a plausible explanation for the antidipsogenic effect of SNP on AngIIinduced water intake (Nicolaidis and Fitzsimons, 1975) without the necessity of assuming opposite mechanical effects caused by the hypotensive and hypertensive effects of SNP and AngII, respectively, on "thirst-neurons" in the SFO.

\section{REFERENCES}

Calapai G, Squadrito F, Altavilla D, Zingarelli B, Campo GM, Cilia M, Caputi AP (1992) Evidence that nitric oxide modulates drinking behaviour. Neuropharmacology 31:761-764.

Calapai G, Mazzaglia G, Cilia M, Zingarelli B, Squadrito F, Caputi AP (1994) Mediation by nitric oxide formation in the preoptic area of endotoxin and tumour necrosis factor-induced inhibition of water intake in the rat. Br J Pharmacol 111:1328-1332.

de Vente J, Steinbusch HW (1992) On the stimulation of soluble and particulate guanylate cyclase in the rat brain and the involvement of nitric oxide as studied by cGMP immunocytochemistry. Acta Histochem 92:13-38.

de Vente J, Schipper J, Steinbusch HW (1989) Formaldehyde fixation of cGMP in distinct cellular pools and their recognition by different cGMP-antisera: an immunocytochemical study into the problem of serum specificity. Histochemistry 91:401-412.

Ehrlich KJ, Fitts DA (1991) Atrial natriuretic peptide in the subfornical organ reduces drinking induced by angiotensin or in response to water deprivation. Behav Neurosci 104:365-372.

Felix D, Akert K (1974) The effect of angiotensin II on neurons of the cat subfornical organ. Brain Res 76:350-353.

Ferguson AV, Day TA, Renaud LP (1984) Subfornical organ stimulation excites paraventricular neurons projecting to dorsal medulla. Am J Physiol 247:R1088-R1092.

Fitzsimons JT (1979) The physiology of thirst and sodium appetite. Monogr Physiol Soc 1:572.

Fitzsimons JT (1980) Angiotensin stimulation of the central nervous system. Rev Physiol Pharmacol 87:117-167.

Garthwaite J, Boulton CL (1995) Nitric oxide signaling in the central nervous system. Annu Rev Physiol 57:683-706.

Harris PJ, Thomas D, Morgan TO (1987) Atrial natriuretic peptide inhibits angiotensin-stimulated proximal tubular sodium and water reabsorption. Nature 326:697-698.

Hattori Y, Kasai M, Uesugi S, Kawata M, Yamashita H (1988) Atrial natriuretic polypeptide depresses angiotensin II induced excitation of neurons in the rat subfornical organ in vitro. Brain Res 443:355-359.

Hope BT, Michael GJ, Knigge KM, Vincent SR (1991) Neuronal NADPH diaphorase is a nitric oxide synthase. Proc Natl Acad Sci USA 88:2811-2814.

Ishibashi S, Nicolaidis S (1981) Hypertension induced by electrical stimulation of the subfornical organ (SFO). Brain Res Bull 6:135-139.

Jurzak M, Schmid HA, Gerstberger R (1994) NADPH-diaphorase staining and NO-synthase immunoreactivity in circumventricular organs of the rat. In: Integrative and cellular aspects of autonomic functions: temperature and osmoregulation (Pleschka K, Gerstberger R, eds), pp 451-459. Paris: John Libbey Eurotext. 
Kleinert H, Maack T, Atlas S, Januszewitcz A, Sealey JE, Laragh JH (1984) Atrial natriuretic factor inhibits angiotensin-, norepinephrine-, and potassium-induced vascular contractility. Hypertension 6[Suppl 1]:I143-I147.

Li Z, Ferguson AV (1993) Angiotensin II responsiveness of rat paraventricular and subfornical organ neurons in vitro. Neuroscience 55:197-207.

McKinley MJ, McAllen RM, Mendelsohn FAO, Allen AM, Chai SY, Oldfield BJ (1990) Circumventricular organs: neuroendocrine interfaces between the brain and the hemal milieu. Front Neuroendocrinol 11:91-127.

Mendelsohn FA, Quirion R, Saavedra JM, Aguilera G, Catt KJ (1984) Autoradiographic localization of angiotensin II receptors in rat brain. Proc Natl Acad Sci USA 81:1575-1579.

Murad F (1994) Regulation of cytosolic guanylyl cyclase by nitric oxide: the NO-cyclic GMP signal transduction system. Adv Pharmacol 26:19-33.

Nicolaidis S, Fitzsimons JT (1975) La dependance de la prise d'eau induit par l'angiotensin II envers la fonction vasomotorice cerebrale locale chez le rat. CR Acad Soc Paris 281:1417-1420.

Pehl U, Schmid HA (1997) Electrophysiological responses of neurons in the rat spinal cord to nitric oxide. Neuroscience, in press.

Phillips MI (1987) Functions of angiotensin in the central nervous system. Annu Rev Physiol 49:413-435.
Phillips MI, Felix D (1976) Specific angiotensin II receptive neurons in the cat subfornical organ. Brain Res 109:531-540.

Saavedra JM (1992) Brain and pituitary angiotensin. Endocr Rev 13:329-380.

Schmid HA, Simon E (1992) Effect of angiotensin II and atrial natriuretic factor on neurons in the subfornical organ of ducks and rats in vitro. Brain Res 588:324-328.

Schmid HA, Schäfer F, Simon E (1995) Opposite effects of angiotensin II and nitric oxide on neurons in the duck subfornical organ. Neurosci Lett 187:149-152.

Simon E, Gerstberger R, Gray DA (1992) Central nervous angiotensin II responsiveness in birds. In: Progress in neurobiology, Vol 39 (Kerkut GA, ed), pp 179-207. Oxford: Pergamon.

Smith PM, Beninger RJ, Ferguson AV (1995) Subfornical organ stimulation elicits drinking. Brain Res Bull 38:209-213.

Southam E, Garthwaite J (1993) The nitric oxide-cyclic GMP signaling pathway in rat-brain. Neuropharmacology 32:1267-1277.

Vincent SR, Hope BT (1992) Neurons that say NO. Trends Neurol Sci 15:108-113.

Wright JW, Harding JW (1992) Regulatory role of brain angiotensins in the control of physiological and behavioral responses. Brain Res 17: 227-262. 